\title{
An Effective Detection of Inrush and Internal Faults in Power Transformers Using Bacterial Foraging Optimization Technique
}

\author{
M. Gopila ${ }^{*}$, I. Gnanambal ${ }^{2}$ \\ ${ }^{1}$ Department of Electrical and Electronics Engineering, Sona College of Technology, Salem, India \\ ${ }^{2}$ Department of Electrical and Electronics Engineering, Government College of Engineering, Salem, India \\ Email: "greatgee@gmail.com, ignan1960@gmail.com
}

Received 22 March 2016; accepted 29 March 2016; published 15 June 2016

Copyright (C) 2016 by authors and Scientific Research Publishing Inc.

This work is licensed under the Creative Commons Attribution International License (CC BY).

http://creativecommons.org/licenses/by/4.0/

(c) (i) Open Access

\section{Abstract}

Power transformers in transmission network are utilized for increasing or decreasing the voltage level. Power Transformers fail to connect directly to the consumers that result in the less load fluctuations. Power transformer operation under any abnormal condition decreases the lifetime of the transformer. Power Transformer protection from inrush and internal fault is critical issue in power system because the obstacle lies in the precise and swift distinction between them. Due to the limitation of heterogeneous resources, occurrence of fault poses severe problem. Providing an efficient mechanism to differentiate between faults (i.e. inrush and internal) is the key for efficient information flow. In this paper, the task of detecting inrush and internal fault in power transformers is formulated as an optimization problem which is solved by using Hyperbolic S-Transform Bacterial Foraging Optimization (HS-TBFO) technique. The Gaussian Frequencybased Hyperbolic S-Transform detects the faults at much earlier stage and therefore minimizes the computation cost by applying Cosine Hyperbolic S-Transform. Next, the Bacterial Foraging Optimization (BFO) technique has been proposed and has demonstrated the capability of identifying the maximum number of faults covered with minimum test cases and therefore improving the fault detection efficiency in a wise manner. The HS-TBFO technique is evaluated and validated in various simulation test cases to detect inrush and internal fault in a significant manner. This HS-TBFO technique is investigated based on three phase power transformer embedded in a power system fed from both ends. Results have confirmed that the HS-TBFO technique is capable of categorizing the inrush and internal faults by identifying maximum number of faults with minimum computation cost as compared to the state-of-the-art works.

\footnotetext{
${ }^{*}$ Corresponding author.

How to cite this paper: Gopila, M. and Gnanambal, I. (2016) An Effective Detection of Inrush and Internal Faults in Power Transformers Using Bacterial Foraging Optimization Technique. Circuits and Systems, 7, 1569-1580. 


\section{Keywords}

\section{Power Transformer, Inrush, Internal Fault, Hyperbolic S-Transform, Bacteria Foraging Optimization}

\section{Introduction}

Power transformers are considered as the most critical and expensive component in the power substations. Power transformer operation under any abnormal condition such as inrush or internal faults results in the compromise of the life of the transformer. Therefore, adequate protection has to be provided for identifying and detecting the inrush and internal faults in power transformer at an earlier stage. Many research works have been conducted in the discrimination of inrush and internal faults by search researchers.

Detection and Location of High Impedance Faults (DL-HIF) [1] was performed using power line communication devices resulted in the ability to effectively identify and detect HIFs. The DL-HIF method applied on multiconductor overhead distribution networks to detect the fault rate in an efficient manner. Phase Angle Difference-based Inrush Restraint (PAD-IR) [2] distinguished the inrush current from internal fault current by applying phase shift between the two currents. With this, the relaying decision remains unaffected resulting in the efficient discrimination between the two currents.

Discrete Wavelet Transform [3] was applied to extract transitory features on transient signal analysis to identify the fault. A new algorithm based on Clarke's transform and Discrete Wavelet Transform (DWT) [4] was applied on power transformer to differentiate between external and internal faults. Entropy approach integrated with Artificial Neural Network [5] resulted in the efficient discrimination between inrush and internal faults in an intelligent based network monitoring system.

Simulation of three-phase power transformer was applied using backward and numerical differentiated formula [6] using linear graph theory resulted in the improvement of inrush faults detection. In [7], Feed Forward Back Propagation Neural Network was applied to distinguish between a magnetizing inrush and power transformer internal faults. An algorithm based on Neural Network Principal Component Analysis (NNPCA) was also designed for digital differential protection of power transformer. A state-of-the-art method for fault detection to power system was presented in [8].

In [9], directly coupled voltage-source inverter was applied to remnant flux in the core for the efficient identification of transformer magnetizing of inrush currents. The application of pre-magnetization resulted in the good reduction in the peak magnetizing inrush currents without applying any external circuit. Particle Swarm Optimization [10] techniques with Artificial Neural Network were applied to predict incipient fault. The integration approach resulted in the accuracy of fault being detected. Diagnosis of power transformer faults using Fuzzy ratio method was applied in [11]. This approach diagnosed multiple faults by also improving the accuracy of fault being detected.

Numerical methods [12] for detecting faults in power transformer were applied by employing finite method. An overview of fault detection, fault classification, fault location and fault direction discrimination was presented in [13]. A review of faults [14] in electric propulsion systems was applied to identify the electric and mechanical faults using robust and reliable mechanism. Fault detection in gas sensing technology was presented in [15] using molecular orbital energy. Fault tolerant converters suitable for microgrids was presented [16] using fault tolerant inverters.

In [17], Electro Magnetic Transient Program - Restructured Version (EMTPRV) model was developed for fault detection and classification in transmission lines. The algorithm in EMTPRV model uses the Wavelet Transform (WT) and Singular Value Decomposition (SVD) for fault detection and classification. Though, the original voltage magnitude of the faulty phases is decreased quickly, but the results are comparatively low in EMTPRV model. With the purpose of detecting bearing faults, a two-stage diagnosis method for fault and fault-severity detections based on the FFT and SVMs is designed in [18]. But, Peak-to-average ratio does not perform optimally. A new detection technique of stator winding faults [19] was introduced in a three-phase PMSM.

In this paper, an effective inrush and internal faults detection mechanism is proposed using Bacterial Foraging Optimization Technique based on Cosine Hyperbolic S-Transform. The proposed Bacterial Foraging Optimiza- 
tion algorithm formulation uses chemotaxis, swarming, reproduction and elimination-dispersion based on foraging strategies to identify and discriminate between inrush and internal faults in power transformer. Initially, the Gaussian Frequency-based Hyperbolic S-Transform is applied in HS-TBFO to detect the faults at much earlier stage which in turn reduces the computation cost. Next, the Bacterial Foraging Optimization (BFO) technique is applied in proposed HS-TBFO for identifying the maximum number of faults covered with minimum test cases which in turn improves the fault detection efficiency in an effective manner.

The remaining of this paper is divided as follows. Section 2 describes the proposed Hyperbolic S-Transform Bacterial Foraging Optimization technique with the aid of the proposed algorithm. Section 3 presents the test results and discussions. The conclusions of this work are presented on Section 4.

\section{Design of Hyperbolic S-Transform Bacterial Foraging Optimization}

In this section, the Design of Hyperbolic S-Transform Bacterial Foraging Optimization technique is developed with the objective of detecting the faults (inrush and internal) at an early stage with reducing the computation cost involved in fault detection. Initially, Gaussian Frequency-based Hyperbolic S-Transform (GFHST) model detects the fault at an early stage by introducing Cosine Hyperbolic S-Transform. Subsequently, Bacterial Foraging Optimization technique is applied in GFHST model to identify the maximum number of faults with minimum test cases.

\subsection{Design of Gaussian Frequency-Based Hyperbolic S-Transform}

The Gaussian Frequency-based Hyperbolic S-Transform (GFHST) model in the proposed technique extracts patterns of inrush faults and internal faults from the captured power transformer. For modeling the Gaussian Frequency-based Hyperbolic S-Transform, modeling of the power transformer is required. The modeling of the power transformer is done by using and combining magnetic and electrical circuits. Figure 1 shows the block diagram of three-phase power transformer.

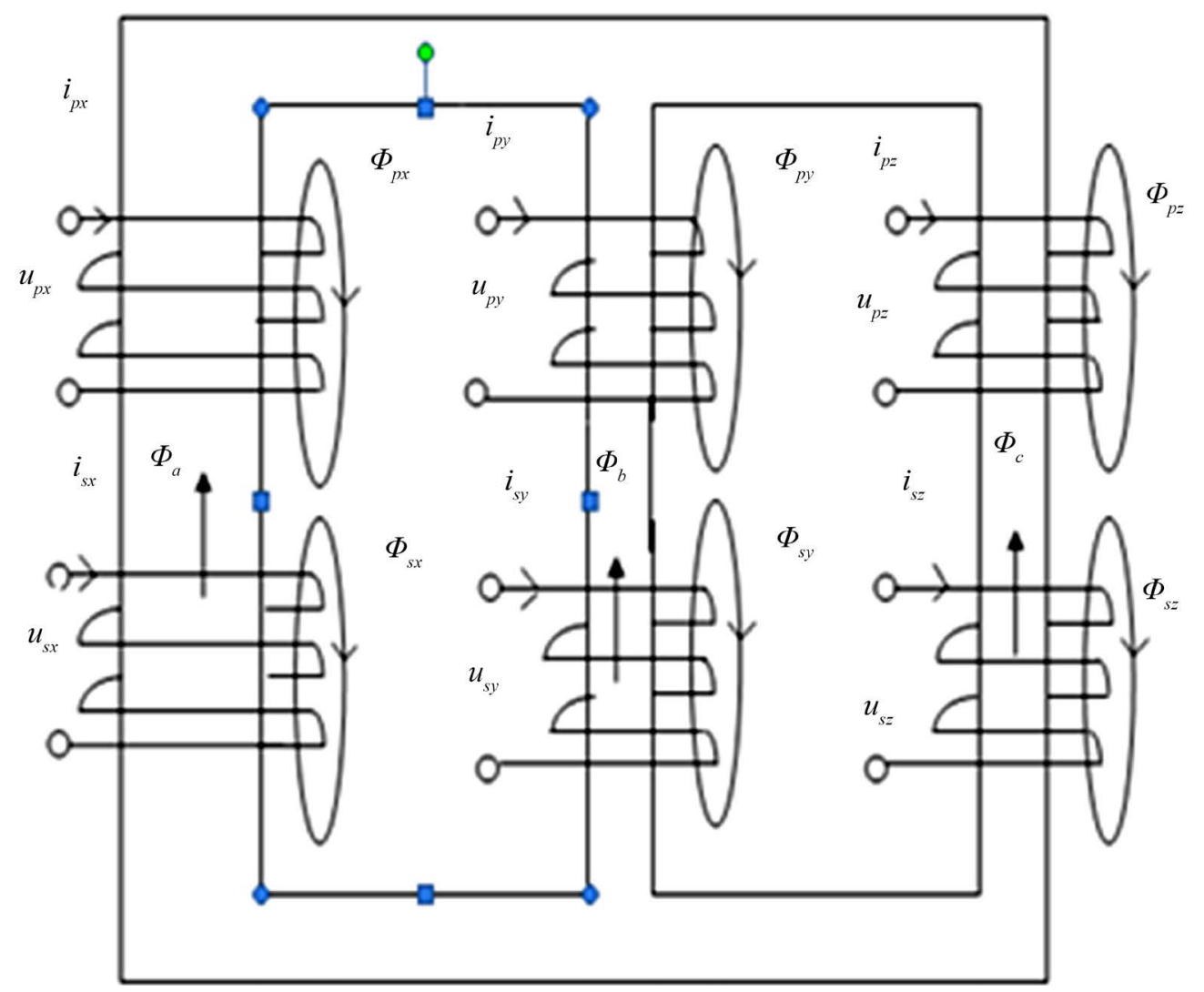

Figure 1. Block diagram of power transformer. 
The GFHST model obtains the information of transient currents based on frequency domain. Followed by this to perform an efficient differentiation between inrush and internal faults, standard deviation is evaluated. The S-Transform is mathematically formulated as given below.

$$
S(f)=\int_{-\infty}^{+\infty} h(f)\left(\frac{f}{\sqrt{2 \pi}}\right) \exp \left\{\left(\frac{-f^{2}}{2}\right)\right\} \exp \{-2 \pi f\} \mathrm{d} f
$$

From Equation (1), $S(f)$ represents the S-Transform of $h(f)$ which is the signal varying with frequency, frequency is denoted by $h(f)$, on the frequency axis. The proposed technique introduced a small variation of Gaussian window with the objective of solving the fault detection at a much earlier stage. The Gaussian S-Transform is mathematically formulated as given below.

$$
G S(f)=\int_{-\infty}^{+\infty} h(f)\left(\frac{f}{\sqrt{2 \pi}}\right) \exp \left\{\left(\frac{-f^{2}}{2}\right)\right\} * G_{f} * \exp \{-2 \pi f\} \mathrm{d} f
$$

From Equation (2), the Gaussian-based S-Transform $G S(f)$ is evaluated using the Gaussian frequency resolution $G_{f}$. With the objective of minimizing the computation while solving fault detection, Gaussian Frequency-based Hyperbolic S-Transform is designed. The Gaussian Frequency-based Hyperbolic S-Transform is constructed using Cosine Hyperbolic S-Transform. The Cosine Hyperbolic S-Transform is mathematically formulated as given below

$$
\operatorname{Cos}(H G S(f))=\int_{-\infty}^{+\infty} h(f)\left(\frac{f}{\sqrt{2 \pi}}\right) \exp \left\{\frac{-f^{2}}{2}\right\}^{1 / 2} * G_{f} * \exp (-2 \pi f)^{-1 / 2} \mathrm{~d} f
$$

From Equation (3), the Cosine Hyperbolic S-Transform $\operatorname{Cos}(H G S(f))$ is measured for minimizing the ripple ratio, which resulting in less computation for fault detection. First, confirm that you have the correct template for your paper size. This template has been tailored for output on the custom paper size $(21 \mathrm{~cm} \times 28.5 \mathrm{~cm})$.

\subsection{Bacterial Foraging Optimization Technique}

Hyperbolic S-Transform Bacterial Foraging Optimization (HS-TBFO) technique used to optimize test suite produced by the application of BFO algorithm that contains possible transmissions and faults. The HS-TBFO technique is applied to produce an Optimal Test by identifying the maximum number of faults covered with minimum test cases. Let us consider the vector array " $V A$ " of " $n$ " elements with progressive phase excitation $[18]$ be formulated as given below.

$$
V A(\theta)=\sum_{i=1}^{n} h_{i}(f) \mathrm{e}^{j(i-1)(k d \cos \theta+\beta)}
$$

From Equation (4), $h_{i}(f)$ are the non-uniform elements (i.e. input signals), with $d$ symbolizing the spacing between the elements and $\beta$ is the progressive phase shift to measure and identify the inrush and internal faults in power transformer. Occurrence of faults in power line transformer results in significant variations causing interruption and delay of power pattern. To locate the occurrence of faults in the vector array " $V A$ ", the proposed HS-TBFO technique measures the degraded array patterns having faulty elements. The " $V A$ " formed using (4) are used to measure the inrush or internal fault by making amplitude excitation equal to zero. This in turn represents the inrush fault element and half of the original excitation to represent an internal fault element.

\subsection{Design of Hyperbolic S-Transform Bacterial Foraging Optimization}

In Hyperbolic S-Transform Bacterial Foraging Optimization technique, a group of E-Coli bacteria or operating conditions (i.e. inrush and internal fault) move in search of rich nutrient concentration. The Bacterial Foraging Optimization technique proceeds by selecting or eliminating bacteria or operating conditions based on their foraging strategies. The natural selection of operating conditions eliminates the signals with poor foraging strategies and favors those having successful foraging strategies. After several cycle's (C) the poor foraging strategies or signals having faults (i.e. inrush or internal fault) are identified and eliminated. The Bacteria Foraging Optimization technique is performed using chemotaxis, swarming, reproduction and elimination-dispersion. 


\subsubsection{Chemotaxis}

The first step in the design of Bacterial Foraging Optimization technique is to detect the movement of inrush and internal fault operating conditions by an activity called chemotaxis. Let us consider " $i^{\text {th }}$ bacterium" (i.e. input signals), with the computational chemotaxis (i.e. computational cost), then the movement of " $i$ th bacterium" is formalized as given below.

$$
R V=\theta^{i}(p+1, q, r)=\theta^{i}(p, q, r)+\operatorname{Step}(i) \frac{\Delta^{t}(i)}{\sqrt{\Delta^{t}(i) * \Delta^{t}(i)}}
$$

From Equation (5), for " $i$ th bacterium", at " $p$ the chemotactic", " $q$ th reproductive" and " $r$ the elimination-dispersal" with Step $(i)$ representing the step size of input signals, the value of " $\Delta$ ", therefore indicates the vector in vector array with random movement specifying the elements either " 0 or 1 " respectively. The resultant value of vector array with random movement is formulated as given below.

$$
R V=V A(\theta)=\{0,1\}
$$

\subsubsection{Swarming}

The second step in the design of Bacterial Foraging Optimization technique is swarming. Swarming analyzes the signal at different frequency bands by cell-to-cell signaling exhibited by bacteria (i.e. input signals) with the objective of detecting and identifying the inrush and internal faults in power line transformer. The cell-to-cell signaling exhibited by bacteria (i.e. input signals) is mathematically represented as given below.

$$
\theta R V(p, q, r)=\sum_{i=1}^{n} \theta \theta^{i}(p, q, r)
$$

From Equation (7), " $n$ " symbolizes the total input signals; " $R V$ " the resultant value of vector array obtained from chemotaxis and $\theta$ representing the search area. Finally, the detection of inrush and internal faults are efficiently made by calculating the standard deviation which clearly distinguishes between inrush and internal faults in power transformer.

$$
\begin{gathered}
S D=\sum_{i=1}^{n} \sqrt{\theta R V_{i}\left(p, q, r^{2}\right)-\theta R V_{i}(p, q, r)^{2}} \\
\text { if } S D>0 \text { then, Detection of inrush else } \\
\text { if } S D<0 \text { then, Detection of internal fault }
\end{gathered}
$$

From Equation (8), the standard deviation " $S D$ " with average resultant value of vector array is denoted by " $\theta R V_{i}$ ". This resultant standard deviation value, classification of inrush and internal fault is made in an efficient manner. Different cycles are applied (i.e. reproduction) for measuring several input signals with the differentiation of inrush and internal fault detection. By applying BFO technique in HS-TBFO, the maximum number of faults is covered with minimum test cases. Figure 2 shows the flow process of Bacteria Foraging Optimization Fault Detection.

Figure 3 shows the algorithmic description of Bacterial Foraging Optimization technique for Fault Detection. As shown in Figure 3, the Bacterial Foraging Optimization Fault Detection algorithm performs three important steps. For each input signals (i.e. bacterium), the cost function is evaluated by an activity called chemotaxis. Followed by this, using resultant value of vector array, cell-to-cell signaling is performed with the objective of detecting the faults (i.e. inrush or internal faults) in the input signals applied on power transformer. Finally, efficient differentiation between inrush and internal faults in power transformer is made by evaluating the standard deviation from the search area. This in turn therefore improves the fault detection efficiency.

\section{Experimental Settings}

Simulation experiments are conducted with Matlab Simulink with a 2000 MVA 220 kV Generator, A 3-phase breaker, a $450 \mathrm{MVA} 220 \mathrm{kV} / 500 \mathrm{kV}$ transformer and a linear load of $250 \mathrm{MW}$ for experimenting the proposed Hyperbolic S-Transform Bacterial Foraging Optimization technique. The sampling rate was $3.2 \mathrm{kHz}$ with each cycle containing 64 samples running for a period of $0.04 \mathrm{~s}$.

The three phase voltage source is connected to the input of the one side and the other side is connected to the 


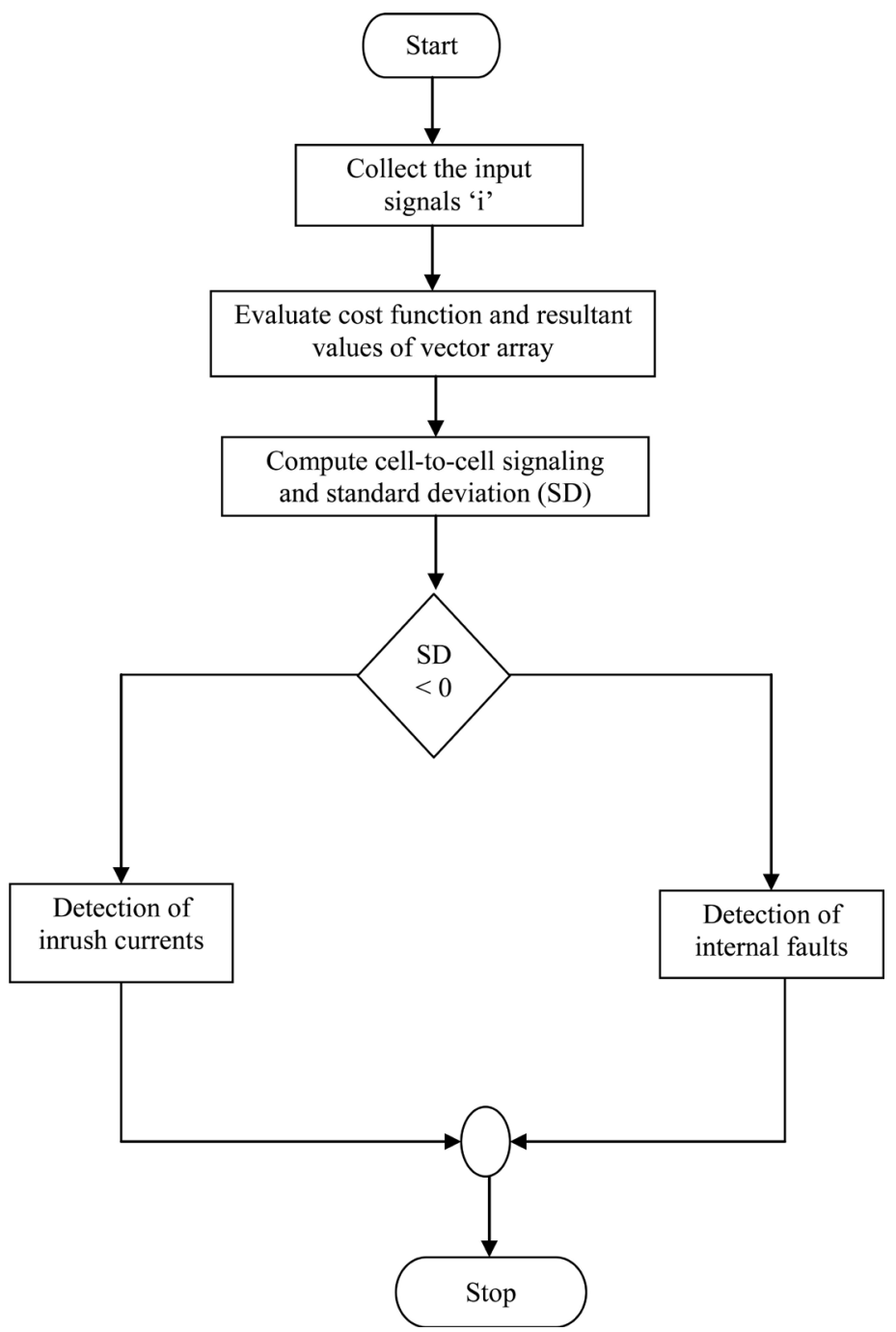

Figure 2. Flowchart for bacteria foraging optimization fault detection algorithm.

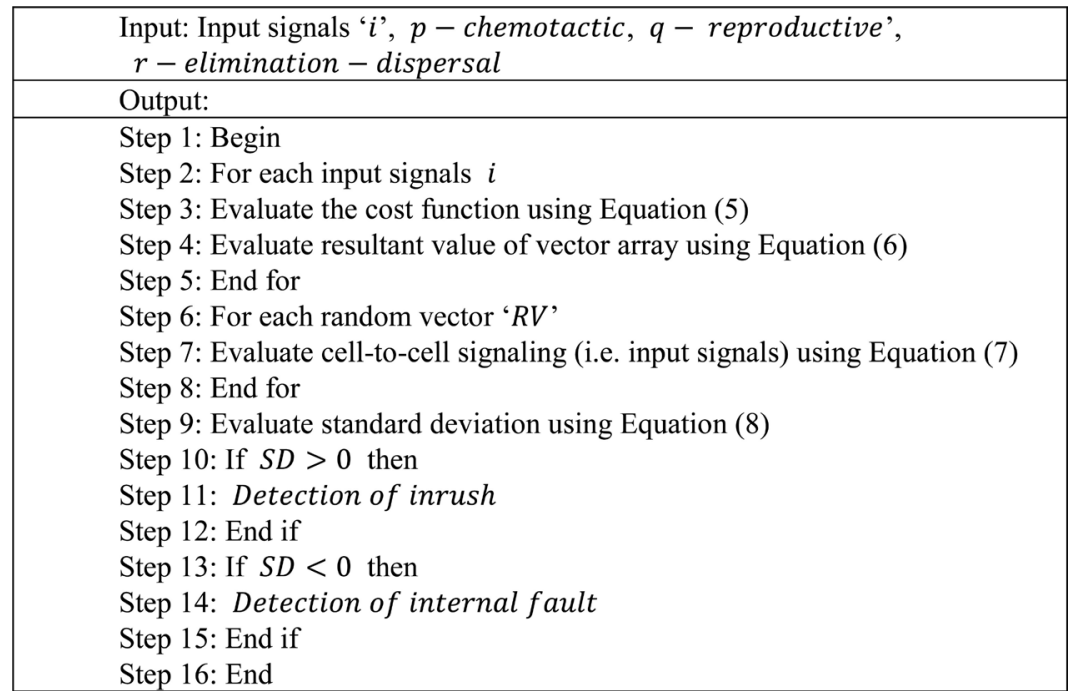

Figure 3. Bacterial foraging optimization fault detection algorithm. 
current measurement block, from which we can measure the Inrush or internal fault current in the power transformer. Here multi winding transformer is taken in which it divided into three phases of $x, y, z$. And the transformer has 20 turns where each of the individual primary and secondary sides of the power transformer for the respective three phases. The HS-TBFO technique is constructed by a PC through a friendly user interface of Matlab coding and comparison is made with existing methods namely Detection and Location of High Impedance Faults (DL-HIF) [1] and Phase Angle Difference-based Inrush Restraint (PAD-IR) [2].

\section{Simulation Results}

The feasible study of the system is done by experimenting through the factors such as fault detection time, fault detection computation cost, number of faults covered and Fault detection on the varying input signals.

Fault detection time (FDT) refers to the time taken to detect the faults (inrush and internal). The fault detection time is measured using the product of number of cycles with respect to time taken to detect fault for each cycle. Fault detection time is mathematically formulated as given below

$$
F D T=\sum_{i=1}^{n} C_{i} * \operatorname{Time}(\text { Fault detection during each cycle) }
$$

From Equation (11), the fault detection time " $F D T$ " is measured with respect to the number of cycles generated $C_{i}$. When lower the fault detection time, more efficient the method is said to be. Computation cost (CC) measures the cost involved in computing the inrush and internal faults. Therefore, computation cost is the product of frequency applied to the fault detection time for varied input signals. The mathematical evaluation of computation cost for computing the inrush and internal faults is as given below.

$$
C C=f * F D T
$$

From Equation (12), the computation cost " $C C$ " is the product of frequency " $f$ " applied during each cycle and the fault detection time. When lower the computation cost, more efficient the method is said to be. Number of faults covered is defined as the ratio of faults identified to the input signals provided as input in power transformer. The number of faults covered is mathematically formulated as given below.

$$
C=\left(\frac{\text { Fault identified }}{\text { Input signals }}\right) * 100
$$

From Equation (13), when higher the faults covered " $F C$ ", more efficient the method is said to be. The performance indices for the fault detection time with respect to varying cycles are shown in Figure 4. These statistical performance indices of fault detection time gives a clear picture of performance improvement for HS-TBFO technique when compared to DL-HIF and PAD-IR and is lower than DL-HIF and PAD-IR showing that HS-TBFO technique has improved performance results in a steady manner with respect to differing cycles. The quantitative results for these performance indices are given in Table 1.

In order to check the robustness of the proposed Hyperbolic S-Transform Bacterial Foraging Optimization (HS-TBFO) technique, series of cycles is applied to the system. The duration of 14 cycles are applied. It shows that the proposed HS-TBFO technique is comparatively lower than the state-of-the-art works though the maximum fault detection time is at $C=14$, comparatively, optimization of fault detection time achieves at $C=8$, with $11.88 \%$ and $20.60 \%$ improvement compared to DL-HIF [1] and PAD-IR [2].

A comparison of the results shows that HS-TBFO technique has more performance improvement using Gaussian Frequency-based Hyperbolic S-Transform that measures Cosine Hyperbolic S-Transform, therefore, more suitable for the commercial systems with higher input points.

The computation cost of the proposed HS-TBFO technique is analyzed using different frequency rates. Experiments are carried out with the frequency rate $5 \mathrm{~Hz}$ and $35 \mathrm{~Hz}$, and the response of the system is investigated in Table 2. The test bed of the proposed computation cost on varied frequency is depicted in Figure 5 and it includes varied frequency rate. The minimum computation cost of the proposed technique is observed between frequency $5 \mathrm{~Hz}$ and $15 \mathrm{HZ}$ using all the methods. But comparatively, lower with the proposed technique that is measured as $1.65 \mathrm{~ms}$ to $3.91 \mathrm{~ms}$ and it varies according to the frequency rate and Cosine Hyperbolic S-Transform being applied.

The computation cost is given in Figure 5. The minimum computation cost on multiple speeds with varied frequency is $1.65 \mathrm{~ms}$ which reaches to $7.12 \mathrm{~ms}$ respectively as compared with the existing DL-HIF [1] and 
PAD-IR [2] respectively. But the computation cost was found to be 1.83 to $7.27 \mathrm{~ms}$ using DL-HIF and $2.10 \mathrm{~ms}$ to $8.37 \mathrm{~ms}$ when DL-HIF and PAD-IR was applied. So comparatively, the computation cost by applying HS-TBFO is observed to be less. This is because of the application of small variation of Gaussian window,

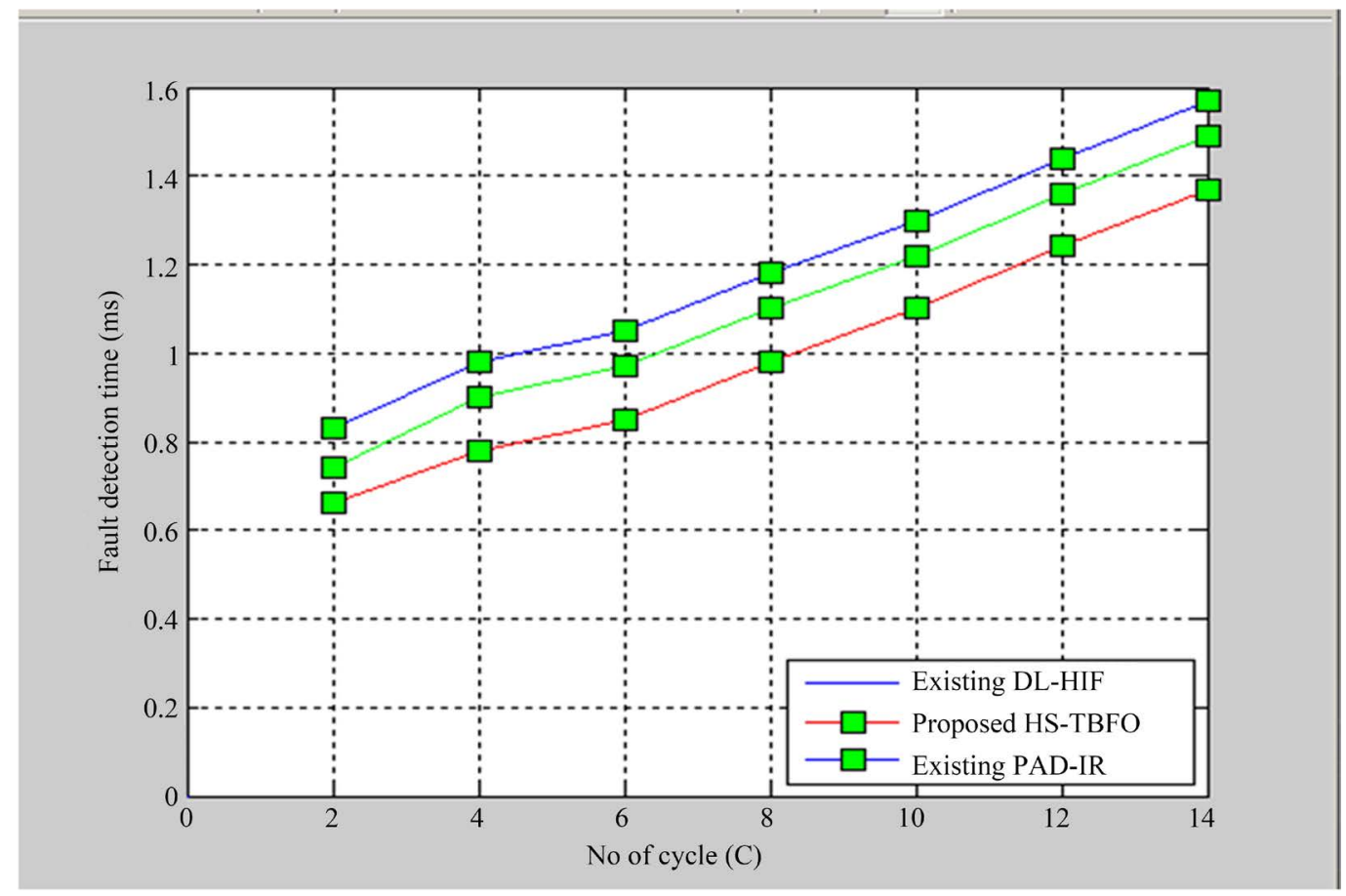

Figure 4. Measure of fault detection time with respect to number of cycles.

Table 1. Comparative result with respect to fault detection time.

\begin{tabular}{cccc}
\hline \multirow{2}{*}{ No of cycles (C) } & \multicolumn{3}{c}{ Fault detection time (ms) } \\
\cline { 2 - 4 } & HS-TBFO & DL-HIF & PAD-IR \\
\hline 2 & 0.66 & 0.74 & 0.83 \\
4 & 0.78 & 0.90 & 0.98 \\
6 & 0.85 & 0.97 & 1.05 \\
8 & 0.98 & 1.10 & 1.18 \\
10 & 1.10 & 1.22 & 1.30 \\
12 & 1.24 & 1.36 & 1.44 \\
14 & 1.37 & 1.49 & 1.57 \\
\hline
\end{tabular}

Table 2. Comparative results with respect to computation cost.

\begin{tabular}{llll}
\hline \multirow{2}{*}{ Frequency $(\mathrm{Hz})$} & \multicolumn{3}{l}{ Computation cost $(\mathbf{m s})$} \\
\cline { 2 - 4 } & HS-TBFO & DL-HIF & PAD-IR \\
\hline 5 & 1.65 & 1.83 & 2.10 \\
10 & 2.85 & 3.00 & 4.10 \\
15 & 3.91 & 4.06 & 5.16 \\
20 & 5.32 & 5.47 & 6.57 \\
25 & 4.12 & 4.27 & 5.37 \\
30 & 6.05 & 6.20 & 7.30 \\
35 & 7.12 & 7.27 & 8.37 \\
\hline
\end{tabular}




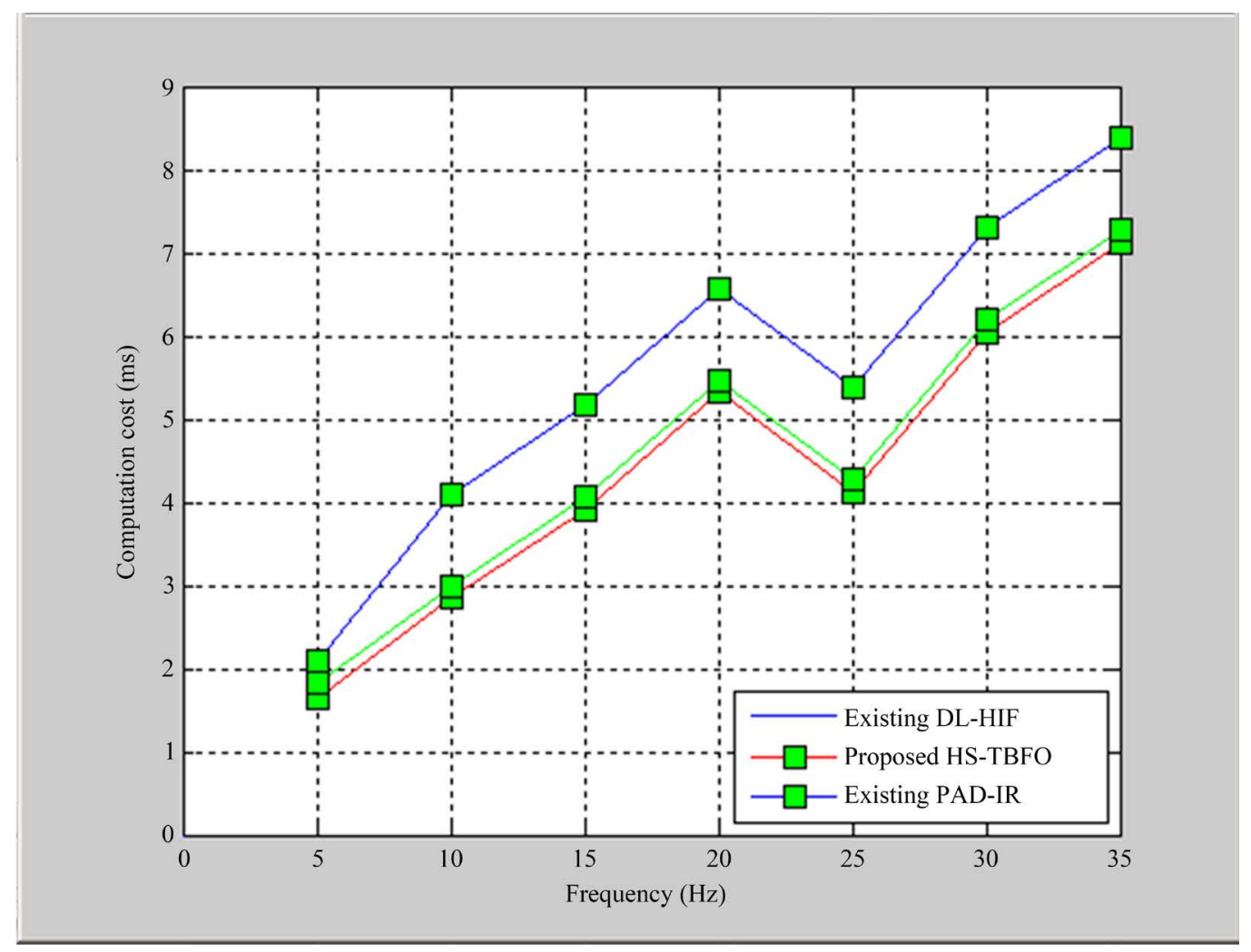

Figure 5. Measure of computation cost with respect to frequency.

Table 3. Comparative result with respect to number of faults covered.

\begin{tabular}{cccc}
\hline \multirow{2}{*}{ Input signals } & \multicolumn{3}{c}{ Number of faults covered (\%) } \\
\cline { 2 - 4 } & HS-TBFO & DL-HIF & PAD-IR \\
\hline 6 & 68.35 & 53.56 & 35.13 \\
12 & 71.13 & 60.10 & 48.05 \\
18 & 74.37 & 63.34 & 51.29 \\
24 & 77.54 & 66.51 & 54.46 \\
30 & 81.13 & 70.10 & 58.05 \\
36 & 82.25 & 71.22 & 59.17 \\
42 & 85.58 & 74.55 & 62.50 \\
\hline
\end{tabular}

Gaussian Frequency-based Hyperbolic S-Transform making an inference of 4.43\% and 27.87\% better comparatively to the two other existing methods [1] [2]. It is therefore significant that the proposed HS-TBFO technique minimizes the fault detection computation cost in an appropriate and flexible manner.

Table 3 summarizes the simulation results of number of faults covered for the proposed HS-TBFO technique and elaborate comparison is made with the existing methods DL-HIF [1] and PAD-IR [2] respectively with respect to differing input signals between 6 and 42 respectively.

To increase the number of faults covered with minimum test cases, in this work Hyperbolic S-Transform is integrated with Bacterial Foraging Optimization. HS-TBFO technique improved the faults covered from 68.35\% to $85.58 \%$ using $\mathrm{BFO}$ algorithm. This helps in improving the faults covered with minimum test cases as shown in Figure 6. The Bacterial Foraging Optimization technique using chemotaxis, swarming, reproduction and elimination-dispersion to identify the occurrence of faults in power line transformer applying step by step procedure of computational chemotaxis, in vector array with random movement specifying the elements either " 0 or 1 " respectively. 


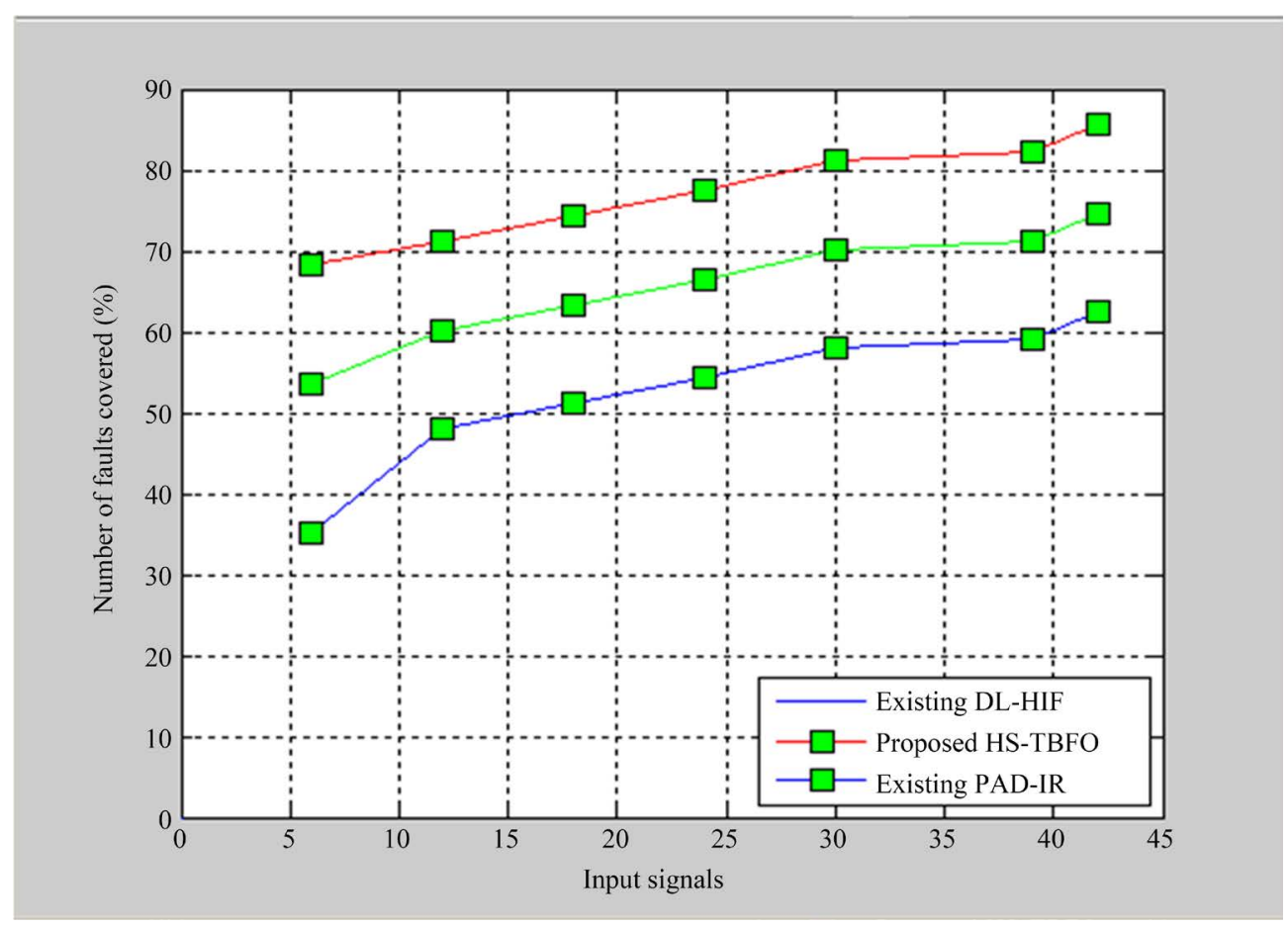

Figure 6. Measure of number of faults covered with respect to different input signals.

Table 4. Comparative results of fault detection efficiency.

\begin{tabular}{cc}
\hline Methods & Fault detection efficiency (\%) \\
\hline HS-TBFO & 73.13 \\
DL-HIF & 65.29 \\
PAD-IR & 59.35 \\
\hline
\end{tabular}

The number of faults covered saw a steady change with maximum number of faults covered when applied with 42 signals (Figure 5). The results showed a good agreement with the peak faults covered, which indicate that the performance of the HS-TBFO technique was comparatively better than the existing DL-HIF [1] and PAD-IR [2] respectively.

Table 4 and Figure 7 show that the proposed HS-TBFO technique provides higher amount of fault detection efficiency on varying input signals with respect to different frequency in the range of $5 \mathrm{~Hz}$ to $35 \mathrm{~Hz}$ when compared to the existing DL-HIF [1] and PAD-IR [2].

The fault detection efficiency is improved because of the application of Bacterial Foraging Optimization. Fault Detection algorithm in HS-TBFO technique. It efficiently estimates the inrush and internal faults in power transformer using the condensed rule based on the cell-to-cell signaling that manages the input signals applied on power transformer for various cycles which in turn improves the fault detection efficiency on varying input signals by $10.72 \%$ and $9.09 \%$ compared to DL-HIF [1] and PAD-IR [2] respectively.

\section{Conclusion}

This research provides an insight into the study of optimization problem used for detecting inrush and internal fault in power transformers using a technique called Hyperbolic S-Transform Bacterial Foraging Optimization (HS-TBFO). The integrated framework Gaussian Frequency-based Hyperbolic S-Transform and Bacterial Foraging Optimization (BFO) technique is combined with Cosine Hyperbolic S-Transform that efficiently helps in handling multiple input signals with varying frequency to minimize the computation cost on power transformer detecting the inrush, an internal fault at an early stage. A prototype of the multiple input signals with different cycles and frequency rate was simulated and tested. A MATLAB environment with Simulink was used to 


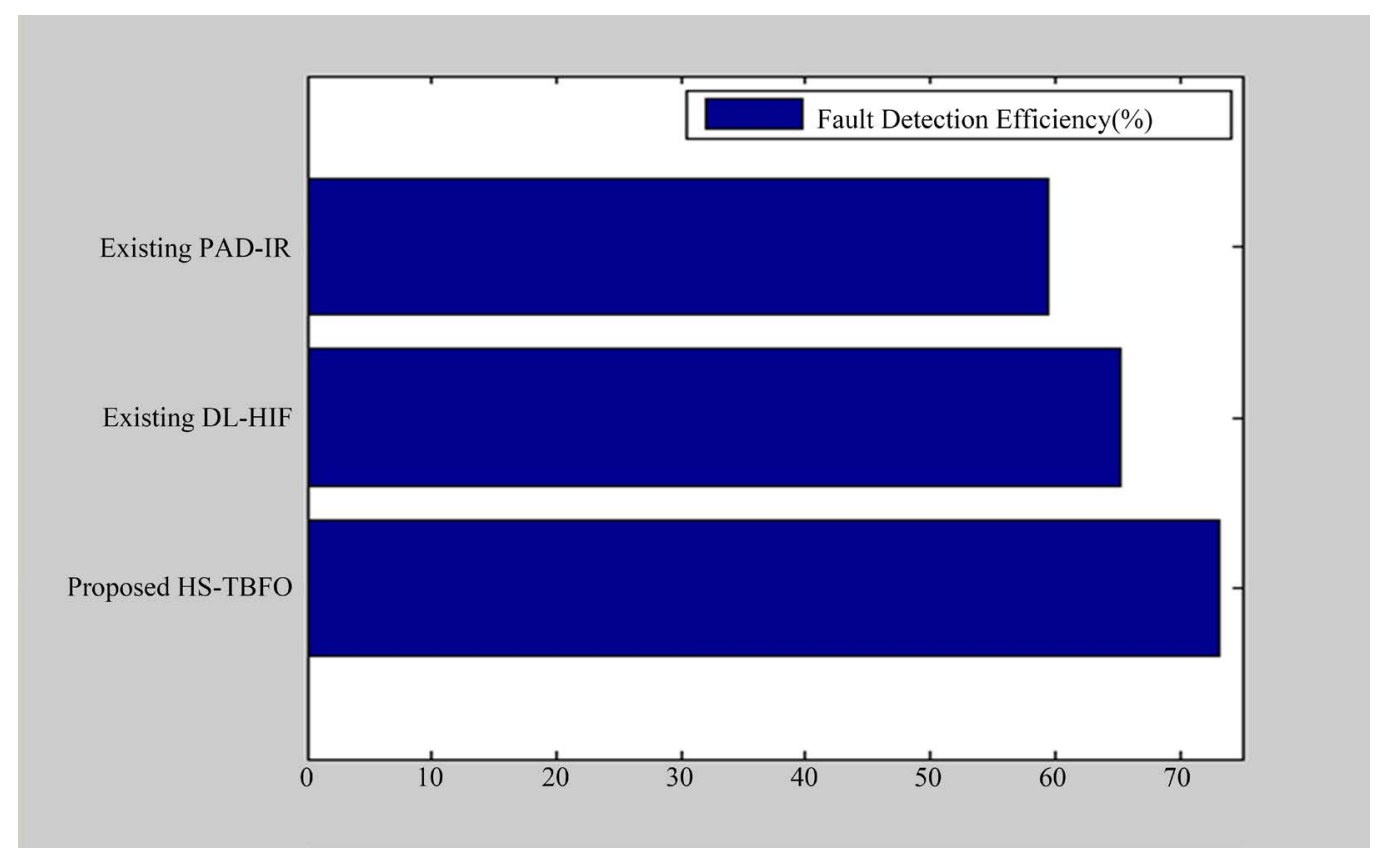

Figure 7. Measure of fault detection efficiency.

calculate the effective fault detection time on varying number of cycles followed by computation cost and fault detection efficiency. The technique also covered maximum number of faults with minimum test cases proving the efficiency of the technique. A simulation result showed the computation cost was reduced by $16.15 \%$ on frequency $5 \mathrm{~Hz}$ to $35 \mathrm{~Hz}$. The transient response test showed that the number of faults covered efficiency was improved by $23.67 \%$ on multiple speeds for varying input signals. The experimental measurements show that the fault detection efficiency varies from $9.09 \%$ to $10.72 \%$ when compared to the state of the art works. Experiments results indicate that the proposed HS-TBFO is an efficient technique that minimizes the computation cost among various input signals in order to efficiently detect the inrush and internal faults in power transformer as compared to the state of the art works.

\section{References}

[1] Milioudis, A.N., Andreou, G.T. and Labridis, D.P. (2015) Detection and Location of High Impedance Faults in Multi Conductor Overhead Distribution Lines Using Power Line Communication Devices. IEEE Transactions on Smart Grid, 6, 894-902. http://dx.doi.org/10.1109/TSG.2014.2365855

[2] Hosny, A. and Sood, V.K. (2014) Transformer Differential Protection with Phase Angle Difference Based Inrush Restraint. Electric Power Systems Research, 115, 57-64. http://dx.doi.org/10.1016/j.epsr.2014.03.027

[3] Oliveira, M.O., Bretas, A.S. and Ferreira, G.D. (2014) Adaptive Differential Protection of Three-Phase Power Transformers Based on Transient Signal Analysis. Electrical Power and Energy Systems, 57, 366-374. http://dx.doi.org/10.1016/j.ijepes.2013.12.013

[4] Noshad, B., Razaz, M. and Seifossadat, S.G. (2014) A New Algorithm Based on Clarke's Transform and Discrete Wavelet Transform for the Differential Protection of Three-Phase Power Transformers Considering the Ultra-Saturation Phenomenon. Electric Power Systems Research, 110, 9-24. http://dx.doi.org/10.1016/j.epsr.2014.01.001

[5] Ozgonenel, O. and Karagol, S. (2014) Transformer Differential Protection Using Wavelet Transform. Electric Power Systems Research, 114, 60-67. http://dx.doi.org/10.1016/j.epsr.2014.04.008

[6] Tokic, A., Milardic, V., Uglesic, I. and Jukan, A. (2015) Simulation of Three-Phase Transformer Inrush Currents by Using Backward and Numerical Differentiation Formulae. Electric Power Systems Research, 127, 177-185. http://dx.doi.org/10.1016/j.epsr.2015.05.020

[7] Tripathy, M. (2012) Power Transformer Differential Protection Based on Neural Network Principal Component Analysis, Harmonic Restraint and Park's Plots. Advances in Artificial Intelligence, 2012, Article ID: 930740. http://dx.doi.org/10.1155/2012/930740

[8] Bunnoon, P. (2013) Fault Detection Approaches to Power System: State-of-the-Art Article Reviews for Searching a 
New Approach in the Future. International Journal of Electrical and Computer Engineering (IJECE), 3, 553-560. http://dx.doi.org/10.11591/ijece.v3i4.3195

[9] Ekström, R., Apelfröjd, S. and Leijon, M. (2013) Transformer Magnetizing Inrush Currents Using a Directly Coupled Voltage-Source Inverter. ISRN Electronics, 2013, Article ID: 361643. http://dx.doi.org/10.1155/2013/361643

[10] Illias, H.A., Chai, X.R., Bakar, A.H.A. and Mokhlis, H. (2015) Transformer Incipient Fault Prediction Using Combined Artificial Neural Network and Various Particle Swarm Optimization Techniques. PLoS ONE, 10, e0137038. http://dx.doi.org/10.1371/journal.pone.0137038

[11] Dhote, N.K. and Helonde, J.B. (2012) Diagnosis of Power Transformer Faults based on Five Fuzzy Ratio Method. WSEAS Transactions on Power Systems, 7, 114-125.

[12] Yan, C.G., Hao, Z.G., Zhang, S., Zhang, B.H. and Zheng, T. (2015) Numerical Methods for the Analysis of Power Transformer Tank Deformation and Rupture Due to Internal Arcing Faults. PLoS ONE, 10, e0133851. http://dx.doi.org/10.1371/journal.pone.0133851

[13] Yadav, A. and Dash, Y. (2014) An Overview of Transmission Line Protection by Artificial Neural Network: Fault Detection, Fault Classification, Fault Location, and Fault Direction Discrimination. Advances in Artificial Neural Systems, 2014, Article ID: 230382. http://dx.doi.org/10.1155/2014/230382

[14] Spyropoulos, D.V. and Mitronikas, E.D. (2013) A Review on the Faults of Electric Machines Used in Electric Ships. Advances in Power Electronics, 2013, Article ID: 216870. http://dx.doi.org/10.1155/2013/216870

[15] Chen, W.G., Zhou, Q., Gao, T.Y., Su, X.P. and Wan, F. (2013) Pd-Doped SnO2-Based Sensor Detecting Characteristic Fault Hydrocarbon Gases in Transformer Oil. Journal of Nanomaterials, 2013, Article ID: 127345. http://dx.doi.org/10.1155/2013/127345

[16] Di Tommaso, A.O., Genduso, F., Miceli, R. and Galluzzo, G.R. (2013) Fault Tolerant Ancillary Function of Power Converters in Distributed Generation Power System within a Micro grid Structure. Advances in Power Electronics, 2013, Article ID: 625130. http://dx.doi.org/10.1155/2013/625130

[17] Lee, J.-W., Kim, W.-K., Oh, Y.-S., Seo, H.-C., Jang, W.-H., Kim, Y.S., et al. (2015) Algorithm for Fault Detection and Classification Using Wavelet Singular Value Decomposition for Wide-Area Protection. Journal of Electrical Engineering and Technology, 10, 729-739. http://dx.doi.org/10.5370/JEET.2015.10.3.729

[18] Hwang, D.-H., Youn, Y.-W., Sun, J.-H., Choi, K.-H., Lee, J.-H. and Kim, Y.-H. (2015) Support Vector Machine Based Bearing Fault Diagnosis for Induction Motors Using Vibration Signals. Journal of Electrical Engineering and Technology, 10, 1558-1565. http://dx.doi.org/10.5370/JEET.2015.10.4.1558

[19] Hadef, M., Djerdir, A., Ikhlef, N., Mekideche, M.R. and N'diaye, A.O. (2015) A Fault Severity Index for Stator Winding Faults Detection in Vector Controlled PM Synchronous Motor. Journal of Electrical Engineering and Technology, 10, 2326-2333. http://dx.doi.org/10.5370/JEET.2015.10.6.2326 